\title{
Augmenting WAMPAC with machine learning tools for early warning and mitigation of blackout events
}

\author{
Sudha Gupta* \\ Electrical Engineering Department, \\ Veermata Jijabai Technological Institute, \\ Mumbai 400 019, India \\ and \\ K.J. Somaiya College of Engineering, \\ Mumbai 400 077, India \\ Email: sudhagupta@somaiya.edu \\ *Corresponding author
}

\section{Faruk Kazi, Sushama Wagh and Navdeep Singh}

Electrical Engineering Department,

Veermata Jijabai Technological Institute,

Mumbai 400 019, India

Email: fskazi@viti.org.in

Email: srwagh@viti.org.in

Email: nmsingh@viti.org.in

\begin{abstract}
The development of phasor measurement unit (PMU) in the power network and availability of real-time communication in wide area monitoring system has enabled the proactive blackout prediction and possibility of mitigation against blackout events. The objective of this paper is to provide a wide area monitoring protection and control (WAMPAC) model which can predict cascade failure and minimise the risk of massive blackout. The proposed model is a combination of simulation and a measurement-based approach. The key contribution of this paper is a topological analysis of grid using graph theoretic approach, blackout prediction using machine learning technique and the mitigation plan against blackout by combining graph theoretic approach and change in voltage phase angle at different buses. The proposed methodology is validated using IEEE 30 bus system.
\end{abstract}

Keywords: blackout prediction; cascade failure; grid topology; neural network; $\mathrm{NN}$; phasor measurement unit; PMU; phasor data concentrator; PDC; probability distribution; wide area monitoring protection and control; WAMPAC.

Reference to this paper should be made as follows: Gupta, S., Kazi, F., Wagh, S. and Singh, N. (2018) 'Augmenting WAMPAC with machine learning tools for early warning and mitigation of blackout events', Int. J. Humanitarian Technology, Vol. 1, No. 1, pp.83-100. 
Biographical notes: Sudha Gupta received her BE in Electronics Engineering from the Government College of Engineering, Ujjain M.P. in 1993, and ME in Electronics Engineering from V.J.T.I., Mumbai, India in 2001. Presently, she is pursuing her $\mathrm{PhD}$ degree in the field of the smart grid technologies from V.J.T.I., Electrical Engineering Department, and working as an Associate Professor in K.J. Somaiya College of Engineering Mumbai India. Her research interests include probability and random variables, communication network and machine learning.

Faruk Kazi received his $\mathrm{PhD}$ degree from Systems and Control Engineering of the Indian Institute of Technology (IIT) Bombay, in 2009. He is currently a Professor of Electronics Engineering with the Department of Electrical Engineering, V.J.T.I., Mumbai, India. His research interests include modelling and control of complex and nonlinear dynamical systems, cyber-physical systems and smart grids.

Sushama Wagh received her ME in Electrical Engineering from Mumbai University, India, in 2003 and $\mathrm{PhD}$ degree from The University of Western Australia, Australia, in 2012, in Electrical Engineering. Currently, she is working as an Assistant Professor at Veermata Jijabai Technological Institute, Mumbai, India. Her research interests include electrical machines, power system stability and smart grid.

Navdeep Singh received his $\mathrm{PhD}$ in Electrical Engineering from IIT Bombay. $\mathrm{He}$ is currently working as a Professor, Department of Electrical Engineering Veermata Jijabai Technological Institute (VJTI) Mumbai, India. His research interest is in mathematical sciences and he is actively involved in teaching and research in the areas of nonlinear dynamics, geometric mechanics and control with special emphasis on application to real world problems.

This paper is a revised and expanded version of a paper entitled 'Neural network based early warning system for an emerging blackout in smart grid power networks' presented at Third International Symposium on Intelligent Informatics (ISI'14), co-located with ICACCI'14, Galgotias College of Engineering and Technology, Greater Noida, India, 24-27 September 2014.

\section{Introduction}

Events like cascade failures in power grid pose severe social and economical problems world wide ('The enquiry committee on grid disturbance in Northern region on 30th July 2012 and in Northern, Eastern and North-Eastern region on 31st July 2012', htttp://www.cea.nic.in/reports/articles/god/grid_disturbance_report.pdf; Andersson et al., 2005). Early detection and mitigation of such events is of immense significance for realising smart grid vision. Motivated by this need many researchers have contributed towards highlighting importance of real-time monitoring, analysis and control in complex power grid operation (Zhang et al., 2010; Siano et al., 2012; Lu et al., 2013; Cirio and Lucarella, 211; De La Ree et al., 2010). Resilient grid operation against such cascade failures can create direct economic benefit for customers (Durand, 2013).

The technological advancement in communication, control and computation (Sauter and Lobashov, 2011; Yang et al., 2011; Zhabelova and Vyatkin, 2012) enables the smart grid (Zhang et al., 2010) to utilise the real-time wide area information fully and 
dynamically. Protection and control techniques have been proposed by the researchers to achieve system level objectives such as prediction and mitigation of cascade link failure. The overall framework of future smart transmission system, such as substations, control centres, and transmission networks have been introduced by Li et al. (2010). In smart grid concept, synergetic combination of wide area monitoring system (WAMS), control, and communication technology is named as wide area monitoring protection and control (WAMPAC).

The WAMS provides global information through phasor measurement unit (PMU) which monitor the dynamic properties of the grid such as transmission line, transformer, generators and load buses in real-time. The WAMS global information of rotor angles, generator speeds, voltage magnitudes and phase angles can be utilised to develop a blackout mitigation model along with intelligent control, adaptive protection relays and remedial action scheme such as forced line outage, load shedding, and controlled islanding. Load shedding (Ding et al., 2013; Sun et al., 2011) and controlled islanding (Xu et al., 2011) for preventive action against cascade failure is a matured technology among researchers. In power systems, a WAMS-based adaptive strategy for controlled islanding was introduced by Song et al. (2014). Sun et al. (2011) developed a scheme for controlled power system islanding using synchronised PMU measurements.

The proposed scheme in this paper is divided into three stages. Initially, the grid data is converted from deterministic to probabilistic data for analysis of cascading link failure. In the second stage, a historical blackout database has been created on the basis of probability distribution of power flow from base case to cascading failure. The higher order moments of historical data are used to train an artificial neural network (ANN) in second stage of proposed model. Once ANN is trained it can predict the critical line which can cause cascading failure by providing online data. Based on early warning signal of cascade link failure, a mitigation plan has been proposed in stage three. Where forced line outage plan is used as a preventive action against early warning of cascade failure. Line selection for outage based on the maximum phase angle difference and topological connectivity of the grid.

Key contribution of this paper is to propose a data centric WAMPAC model with an aim of prediction and mitigation against blackout. A neural network (NN)-based early warning system for blackout prediction has been proposed in our earlier attempt (Gupta et al., 2015) where, a machine learning (ML) technique along with probabilistic model (Gupta et al., 2014) was used for prediction of emerging blackout. Prediction was based on offline analysis of past blackout events and online data which is assumed to be available from WAMS. The present paper uses early warning, generated from Gupta et al. (2015), as a precursor of blackout prediction. Based on early warning, a mitigation plan is proposed to protect the grid against blackout. The concept of phase angle difference and the grid topology has been used for faster grid protection.

The existing power system control centres typically performs steady state contingency analysis, where each contingency event is analysed by power flow studies. The proposed prediction and mitigation model are based on simulation and measurement-based approach and can analyse online contingency which is the key characteristic of the smart grid proactive blackout prediction and mitigation planning.

This paper is organised as follows: Section 2 presents proposed WAMPAC methodology for blackout prediction and mitigation. Section 3 is a gist of blackout prediction using ANN ML technique. A blackout mitigation plan proposed in Section 4. 
Section 5 is a case study to validate the mitigation plan where IEEE 30 bus system is used as prototype power system. Some open research issues are discussed in Section 6 along with conclusions.

\section{Proposed methodology}

The traditional supervisory control and data acquisition system (SCADA) is based on steady state power flow analysis, which is unable to observe dynamic characteristics of the power system at a required resolution. Whereas the dynamic measurements become possible with PMUs which precisely monitor the voltage and current phasors through a timing reference generated by global positioning system (GPS).

PMUs at substations measures voltage and current phasors generally at a rate of 20-60 times a second and compute MW/MVAR and frequency in very precise synchronisation, with $\mu$ s accuracy. Hence, they are capable to track grid dynamics in real-time compared to SCADA system.

As shown in Figure 1, we assumed that PMU at substation measures time synchronised dynamical data online and send it to phasor data concentrator (PDC). The PDC gather data from several PMUs with identical time tags, align the time tag and create a record of data from wide area network. Hence, at PDC, PMUs data are available for analysis and processing.

Figure 1 Proposed scheme of WAMPAC system for blackout prediction and mitigation

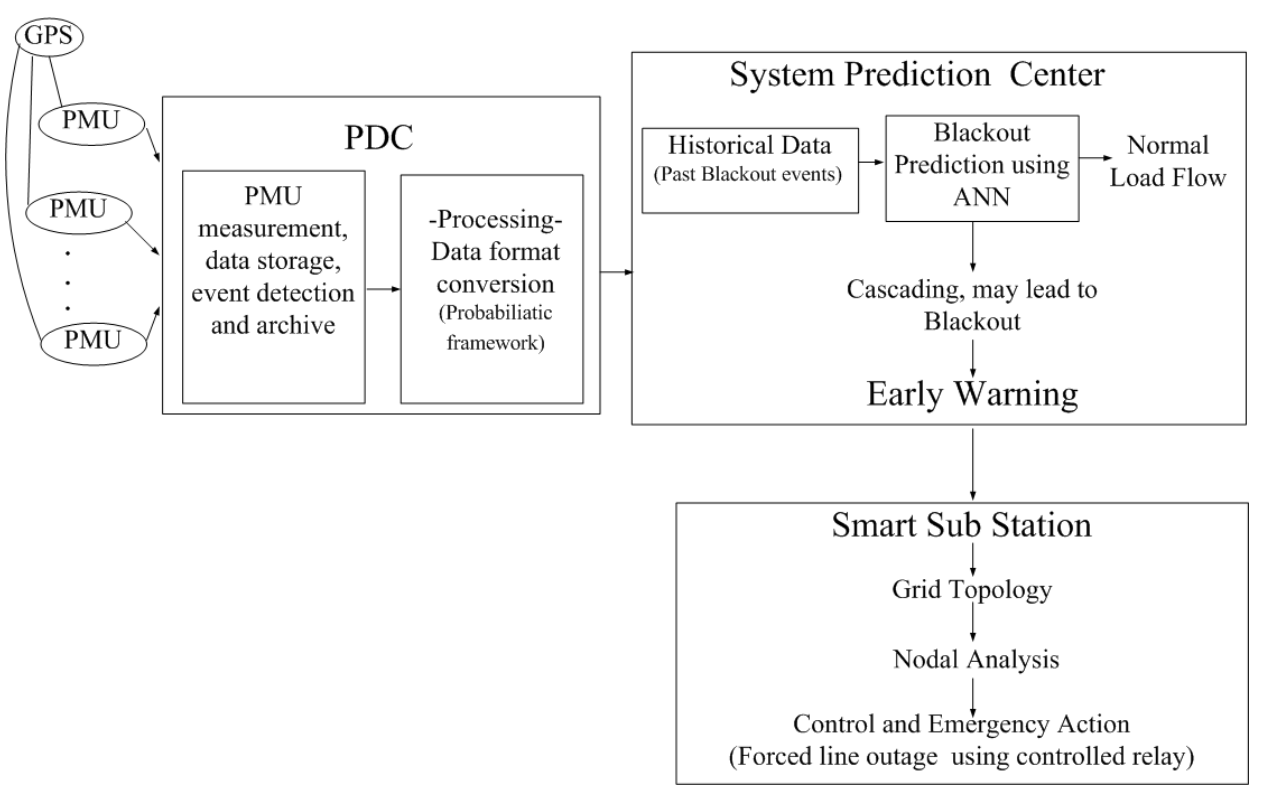

The measured data can be analysed in real-time at control centre PDC where the data format has been changed from deterministic to probabilistic (Gupta et al., 2014) for blackout prediction purpose. 


\subsection{Probabilistic data from Gaussian distribution}

Let $X$ be a random variable which is symmetrically distributed in a Gaussian distribution under normal working of the grid. In static power flow network, parameters can be calculated in terms of mean, variance, standard deviation, probability density function (PDF) and cumulative distribution function (CDF). The mean of $X$ is a measure of the centre of distribution and the variance of $X$ is a second moment of $X$ about the mean which measures the spreads of random variable $X$,

$$
V_{a r}(X)=E\left(\frac{X-\mu}{\sigma}\right)^{2}
$$

where $X$ is a random variable, $\sigma$ is standard deviation of random variable. Variance is $\sigma^{2}$ and $\mu$ is the mean value of the random variable.

Under the steady state mode of normal power grid where all the transmission lines are working within their rated capacity, the Gaussian probability distribution has to be symmetrical about its mean. In dynamical state, power flow is not fixed all the time, it changes with time according to loading. Hence, in dynamical power flow such as cascading link failure, these Gaussian curves start shifting from Gaussian to non-Gaussian distribution (Gupta et al., 2014). In this situation, higher order moments are required to analyse dynamical changes in load flow. The higher order moments have been used in Gupta et al. (2015) to extract the dynamics of cascading link failure. Approximately 45 complete blackout scenario has analysed with probabilistic model and used as historical database. The higher order moments of historical data is used as a feature vector for training and testing of ANN in Gupta $t$ al. (2015).

As shown in Figure 1 at prediction centre, a scaled conjugate gradient (SCG) feedforward supervised ANN is used to train prediction model using historical blackout data (Gupta et al., 2015). Once ANN trained, it classify whether power flow in the grid is normal or blackout may happen by simply giving online input data to ANN model (Gupta et al., 2015).

Precise and accurate historical database is very important and key characteristic for training and testing of ML tools. In WAMPAC, high resolution time synchronised historical PMU data can improve blackout assessment capability and accuracy of historical database. Training of ML with such data sets can further improve accuracy of blackout prediction.

\section{Blackout prediction}

The aim of training the ML strategy is to arrive at a model based on blackout historical data for predicting the blackout by providing online data attributes. As shown in Figure 1 and Figure 2, once ANN trained by historical database it can predict whether the grid is in normal state or blackout may happen by giving only the online data as an input. The early warning information can be generated based on output of SCG-ANN to prepare a mitigation plan against future blackout. 


\subsection{Historical database}

A historical database has been generated from IEEE 30 bus test system using MATLAB and PowerWord simulator. We assumed that grid data monitored by PMU and received at PDC as shown in Figure 2. At PDC, it is first converted into probabilistic data using normal or Gaussian distribution and from the distribution curves the variables such as mean, variance, and higher order moments were calculated. A historical database of normal grid operation as well as blackout case are generated from these variables using probabilistic framework (Gupta et al., 2014) of cascading failure. As shown in Figure 2, higher order moments of various normal and blackout cases are calculated and stored in historical database.

The proposed model requires these historical data as an input to train and validate SCG-ANN for future blackouts prediction. Predicted output is further given to system protection centre for emergency action such as operation of system protective relays, breaker or forced line outage.

Figure 2 ANN prediction model

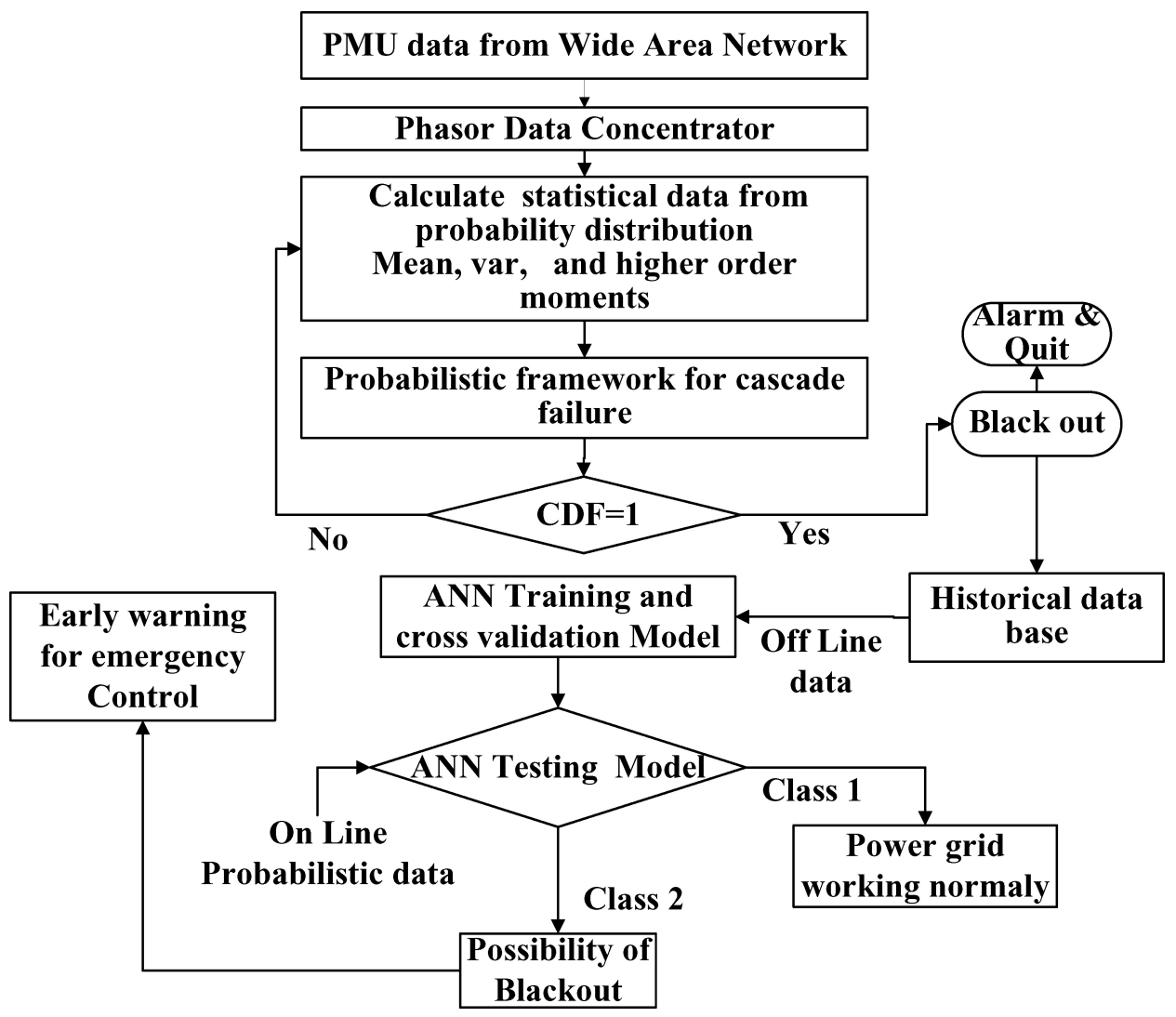

Source: Gupta et al. (2015) 


\subsection{Blackout prediction with SCG-ANN technique}

As shown in Figure 2, the blackout prediction model was proposed in our earlier work (Gupta et al., 2015) using probability theory (Gupta et al., 2014) and SCG-ANN technique to identify critical link, responsible for possible blackout. The paper (Gupta et al., 2015) used ANN ML tool for early warning of blackout where probabilistic feature vector was used for training and testing of ANN.

The input feature vector to ANN was selected from offline historical database (45 blackout cases). Out of 45 blackout cases data, $75 \%$ of the data was used for training $15 \%$ for validation and $10 \%$ data used for testing using feed forward network and fast supervised SCG ANN learning algorithm (Gupta et al., 2015).

\section{Blackout mitigation plan}

The present paper takes early warning signal as an indication for the onset of blackout and proposes a mitigation plan based on grid topology and phasor angle monitoring. Phasor angle analysis at substation PDC identifies high angle displacements, to select suitable line for forced outage. In 2010, Dobson and Parashar, reported in Dobson et al. (2010) that, simulations of the grid just before the Northeast blackout of August 2003 showed an increasing angle differences between the Cleveland and West Mahican, which indicate that large phase angle differences could be an indication of emerging blackout (Cummings, 2005). In WAMPAC system, PMU directly provide the accurate phase angles at the high sub-second rate (100 to 200 samples per second) compare to traditional slow (typically every 5 minutes) state estimation model in SCADA system. Hence, with the use of PMUs in WAMS the most sensitive phase angles can identify in real-time for each iteration of cascading link failure.

Consider a power network where generator and load buses as nodes and transmission lines forming links connecting the nodes as per the network topology as shown in Figure 3. According to Sullivan and Lee (1977) and Wang et al. (2008), the DC power-flow contingency analysis for a load flow from node $i$ to node $j$, the injected active power $p_{i}$ can be expressed by

$$
p_{i}=-p_{j}=\frac{\left|V_{i}\right|\left|V_{j}\right|}{X_{i j}} \sin \left(\delta_{i}-\delta_{j}\right)
$$

where, $\left|V_{i}\right|,\left|V_{j}\right|$ is respective voltage magnitude at node $i$ and $j \cdot p_{i}$ and $p_{j}$ is a injected active power, $X_{i j}$ is an impedance of the transmission line connecting to $i$ and $j . d_{i}$ and $d_{j}$ is a voltage phase angle at node $i$ and $j$, respectively.

For DC power flow, the voltage magnitude at all nodes is maintained at 1 per unit $\mathrm{(pu}$. Further, as the system synchronisation is always maintained under normal operating conditions the angular difference between two neighbouring nodes is very small. Hence, $\left(\delta_{i}-\delta_{j}\right)$ being very small $\sin \left(\delta_{i}-\delta_{j}\right)$ is approximately equal to the angle difference and can be replace by $\left(\delta_{i}-\delta_{j}\right)$ which will modify (2) to

$$
p_{i j}=\frac{\left(\delta_{i}-\delta_{j}\right)}{X_{i j}}
$$


The total load flow from the node $i$ to node $j$ is mathematically represented as

$$
P_{i}=\sum_{j} \frac{\left(\delta_{i}-\delta_{j}\right)}{X_{i j}}
$$

The same can be written in matrix form

$$
\Delta \delta=K \Delta P
$$

where $\Delta d$, represents change in phase angle at system bus due to change in power injection of $\Delta P$ and the $K$ matrix element is a function of line admittance. Hence, in the power grid, we can get bus voltage phase angle as a function of power injection.

According to (5), for a fixed set of power injections $p$, tripping of one line will change both the $\Delta P$ and the $K$ matrix element from their base value. The above equations will provide information of changes in the bus voltage angle due to network topology changes.

Figure 3 Power flow between bus $i$ to bus $j$

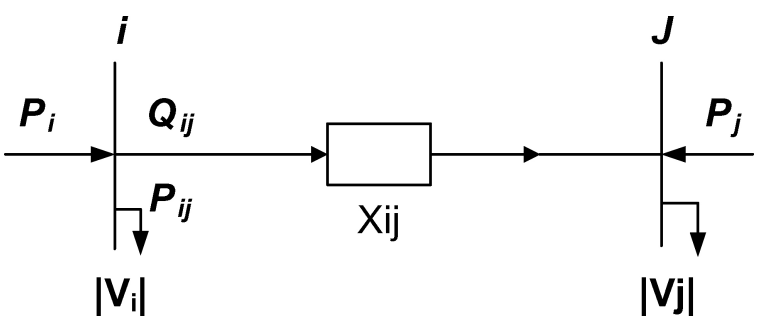

\subsection{Graphical model}

The graph theoretic model consider the generator and load buses as a node (vertices) and transmission line, transformers as a link. In equation (5), $k$ matrix (admittance matrix) represents node to node connectivity. For a fixed set of power injections $P$, if a line or lines are removed, both the $K$ matrix and the $\delta$ vector will change from their base case values (Sullivan and Lee, 1977) by an amount $\Delta K$ and $\Delta \delta$. Hence, topological line state information of $K$ matrix and change in bus angle from normal to cascading state can be calculated at PDC using online PMU data. The relative changes in voltage phase angle can be analysed as;

$$
\Delta \delta=\Delta \delta_{\text {present }}-\Delta \delta_{\text {base }}
$$

At time $t$ for the $m^{\text {th }}$ sample of PMU data the difference in phase angle from the base value can be calculated as:

$$
\Delta \delta=\Delta \delta_{m}-\Delta \delta_{\text {base }}
$$

Here, for mitigation plan the current grid topology information from $K$ matrix can be combined with early warning signal at critical link along with real-time PMU phase angle measurements to identify the critical angle and outage line. Then, these quantities can be monitored, reported and visualised according to dynamic changes in the power system for selection of forced line outage to prevent emerging blackout. 
Forced line outage may result in formation of cluster in the grid. Generators in one cluster may run with over-frequency, while those in other cluster run with under-frequency, sensed by corresponding relays. The temporary survival of the clusters under this condition can be taken care of by either shedding load or providing extra load, as the case may be, till the line between the clusters is restored.

\section{Case study and simulation analysis}

The IEEE 30 bus test-bed system (Gnanadass, 2011) has been built in Powerworld Simulator and used in this paper as a prototype power network. The one line diagram of IEEE 30 bus system and parameters are shown in Figure 4 and Table 1, respectively. To understand the topology of power network a graph theoretic model of test-bed system has been created by graphical model.

Figure 4 One line diagram of IEEE 30 bus test-bed system

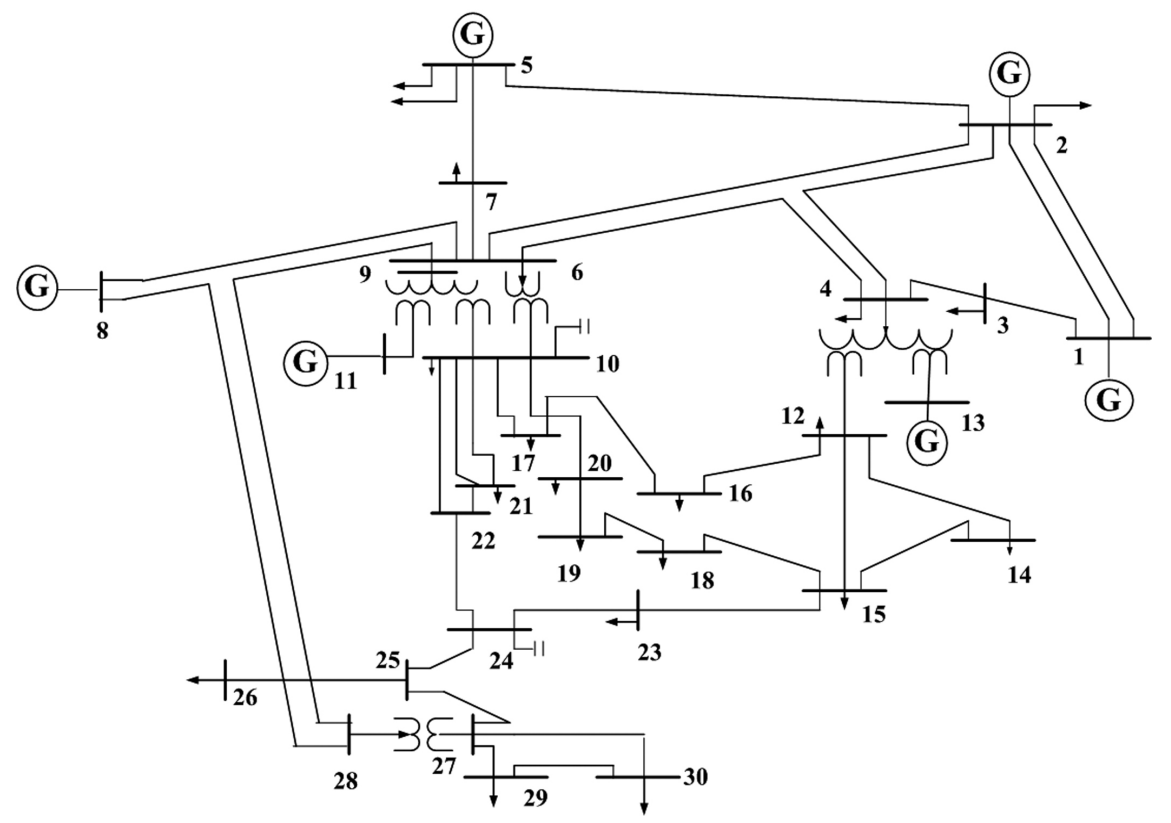

Table 1 IEEE 30 bus test bench parameter

\begin{tabular}{lc}
\hline Parameter & Description \\
\hline No. of buses & 30 \\
Slack bus no. & 1 \\
Generators & 6 \\
Loads & 21 \\
Lines/transformers & 42 \\
Load & $283.4 \mathrm{MW}$ \\
Generation & $289.1 \mathrm{MW}$ \\
\hline
\end{tabular}




\subsection{Graphical model of IEEE 30 bus system}

A model $(G)$ can be represented in the form of nodes and link as

$$
G=(V, E, W)
$$

where $V$, vertices or node (buses) of the graph, $E$ is edges (transmission line or transformers) of the graph and $W$ is the weight function (line impedance) of the edges.

Figure 5 Graphical model of IEEE 30 bus system (base case) (see online version for colours)

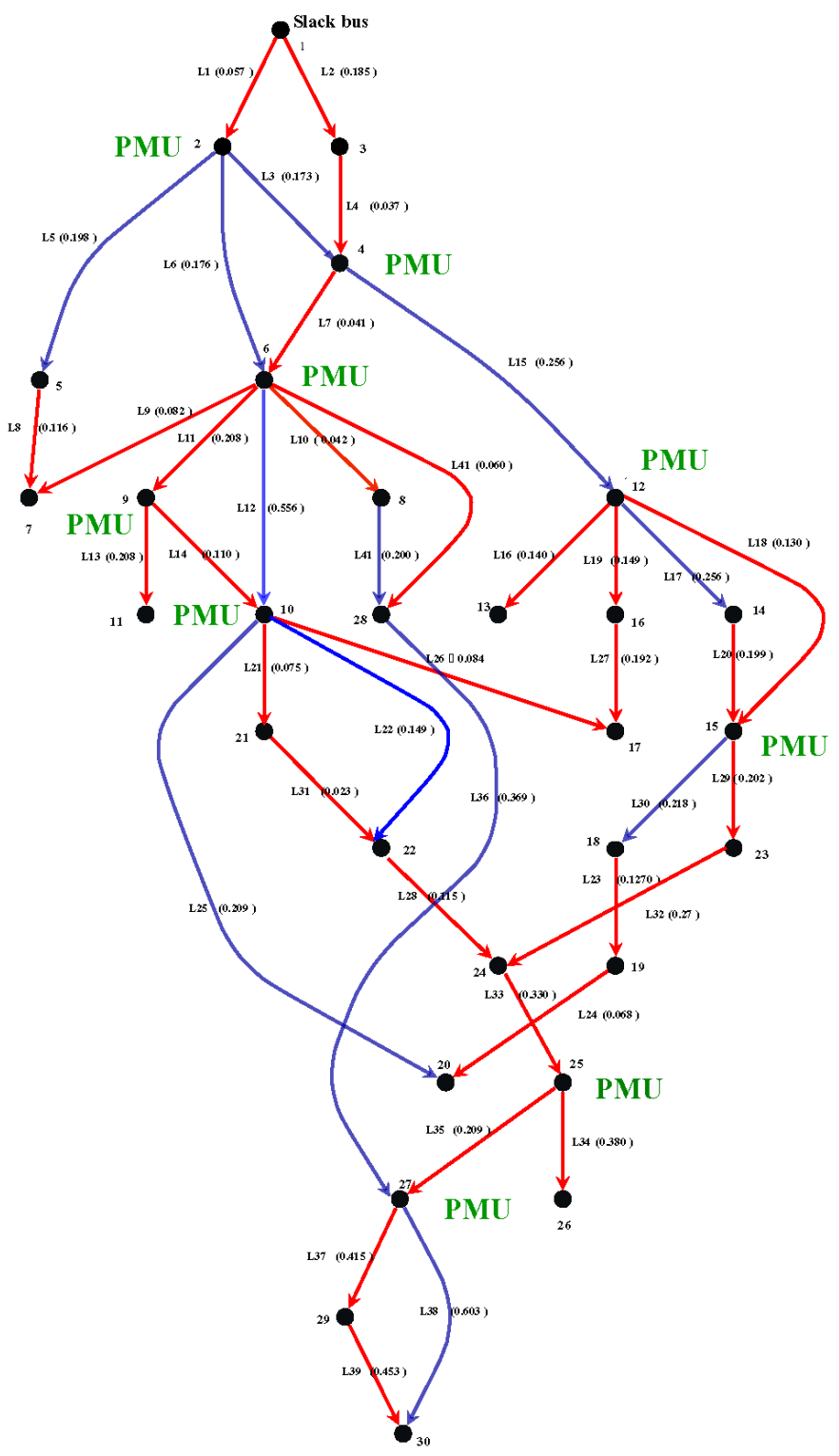


A weighted directed tree $T=(V, E, W)$ of the test-bed system along with maximum capacity link connectivity (red colour link) is shown in Figure 5. The number across transmission line representing the line numbers and values along with lines indicating line impedance. Minimum impedance line carries maximum power flow. Information of maximum power flow links for complete grid helps in contingency analysis of transmission line during cascading failure. As chances of line tripping and amount of power redistribution after line tripping is more with high capacity (low impedance) lines, hence, monitoring of these lines are more important. Keeping this in mind, PMU placement for proposed model considered on minimum spanning tree (MST) algorithm. The MST algorithm gives information of local to global connectivity and provides feasible solution of power flow in the power grid with minimum impedance (maximum power flow) transmission lines of a network.

The MST is a sub-graph of $\mathrm{G}$ and defined as

$$
W(T)=\sum_{I, J \in T} W(I, J)
$$

where $W(T)$ is a shortest path of the grid network and $W(I, J)$ is transmission line impedance between node $I$ and $J$. MST algorithm is used in the literature (Manousakis et al., 2012; Denegri et al., 2002; Kamwa and Grondin, 2002; Nuqui and Phadke, 2005) for optimal PMU placement.

The secure PMU placement for the IEEE 30 bus system according to Kim and Poor (2011) are bus number 2, 4, 6, 9, 12, 10, 15, 25 and 27. MST of IEEE 30 bus system using Kruskals method has been calculated for this paper which is shown in Figure 5 where red lines are showing maximum load bearing lines starting from reference node (slack bus) 1 to all other nodes (buses). Figure 5 contains all $(n-1)$ nodes are showing local to global connectivity of grid without looping. Figure 5, covers all the neighbouring nodes (buses) and provide shortest path to connect other nodes. Hence, we consider the best PMU placement for IEEE 30 bus system (Kim and Poor, 2011) are bus no. 2, 4, 6, 9, $12,10,15,25$ and 27 , for this paper.

\subsection{Nodal analysis and mitigation}

This paper has considered the intimation from early warning (Figure 2) and prepared a forced line outage plan based on voltage phase angle difference, as an emergency action against onset of blackout.

- Under the normal working of the grid load flow on transmission lines are under their rated capacity. This scenario considered as base case (normal) power flow. IEEE 30 bus power system has developed in Powerworld simulator and the same is shown in Figure 6. Figure 6 is a base case scenario. Figure 7 is a graph of voltage phase angles at different buses under base case of the grid, for graph simplicity we have taken absolute phase angle.

- Consider an SCG-ANN-based blackout prediction model for early warning system proposed in the previous paper (Gupta et al., 2015) and shown in Figure 2. Consider at time $t$ an early warning signal indicating onset blackout after tripping of link L22 of test-bed system. As shown in Figure 5, graphically this link is connected between node no. 10 to node no. 22 and closely monitored by PMU installed at node no.10. 
Figure 6 Normal load flow in IEEE 30 bus system (base case) (see online version for colours)

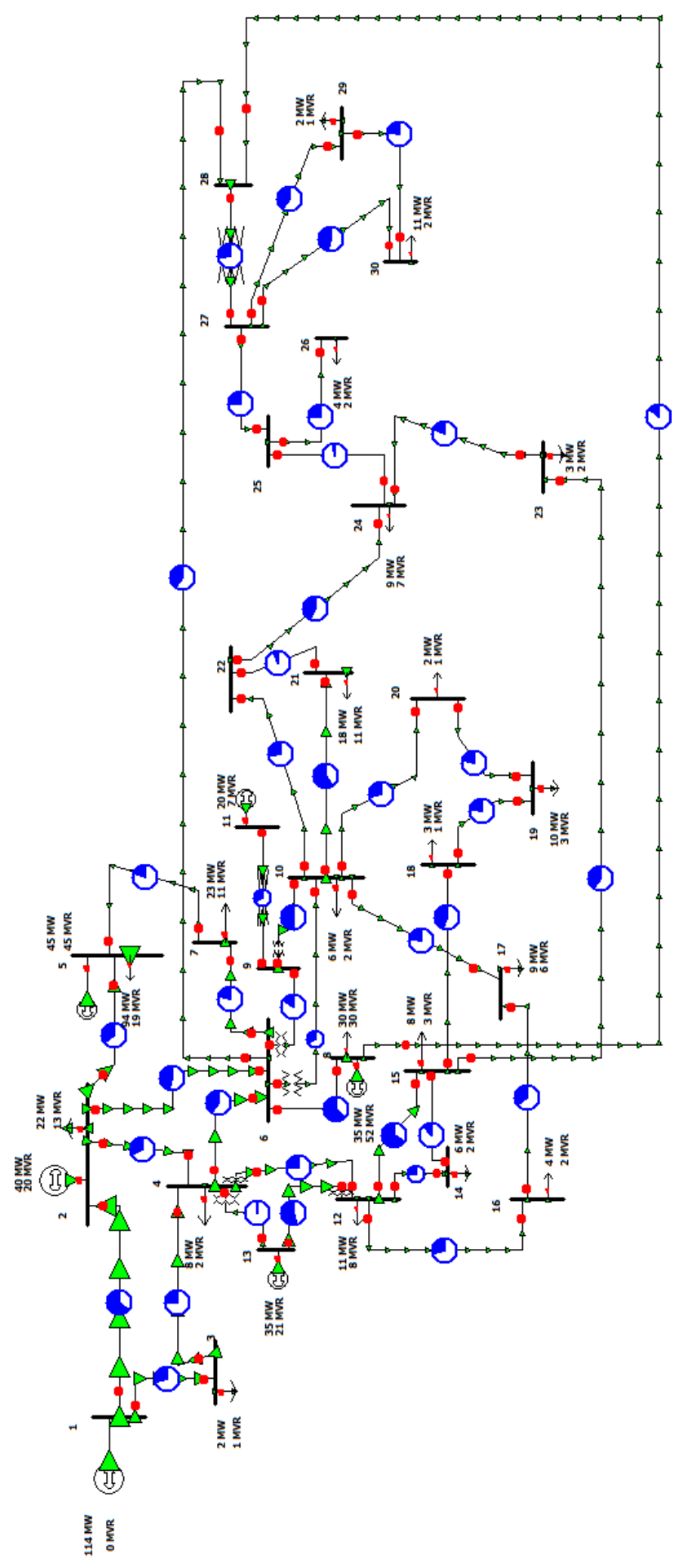


- Figure 8 is a scenario of perturbed grid on receiving of early warning signal which is reflecting the over loaded lines in the grid. The overloaded line is indicated by $\%$ loading with solid circle which is reflecting stress in the grid.

- $\quad$ Analysis of perturbed grid on receive of early warning is shown in Figure 9. Figure 9 is a graph of bus numbers verses phase angle at respective bus. The stress has increased with increase in phase angle difference on the bus from normal grid to perturbed grid. Analysis of Figure 9 shows that maximum phase angle difference from base case (Figure 7) to perturbed case (Figure 9) has occurred on bus number 21 and 22 .

- $\quad$ Power systems under perturbed condition showing large voltage phase angle differences compared to base case and the same is analysed and shown in Figure 9 where marker is indicating maximum phase angle difference on bus 21 and 22 at the time of early warning signal. In WAMPAC system (Figure 1), the PMU monitors and measures these synchrophasor phase angle with time tag and maximum phase angle difference calculated at control centre PDC. Topological analysis from Figure 5 indicating that the link between bus 21 and 22 is L31. Hence, maximum stressed buses can be separated by outage of line L31.

Figure 7 Voltage phase angle corresponds to bus number (base case) (see online version for colours)

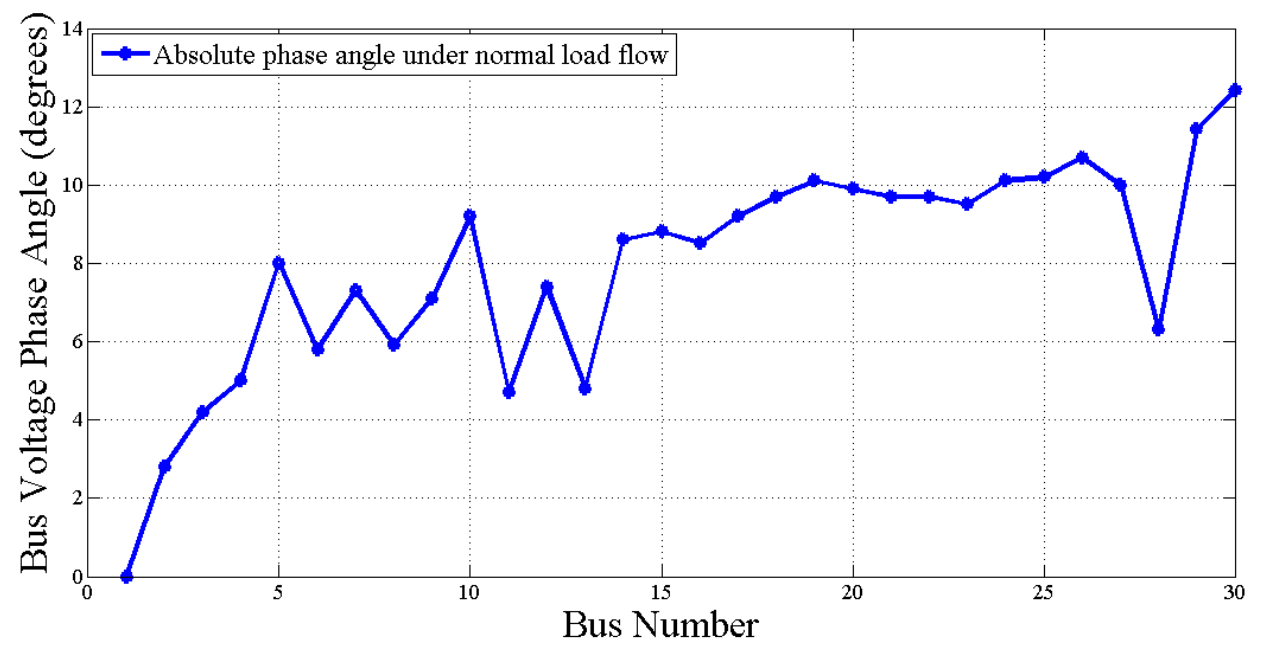

- Figure 10 is a simulation graph showing the power flow on transmission line from base case to perturbed case. Analysis of topological model of IEEE 30 bus system in Figure 5, provided information of bus connectivity.

Phase angle analysis from Figure 9 indicating the highly stressed area of test-bed system, i.e., the buses 21 and 22. Graphically, this can be separated from the entire grid by forced outage of line L31 via protection relay. 
Figure 8 Load flow on onset of early warning (perturbed condition of the grid) (see online version for colours)

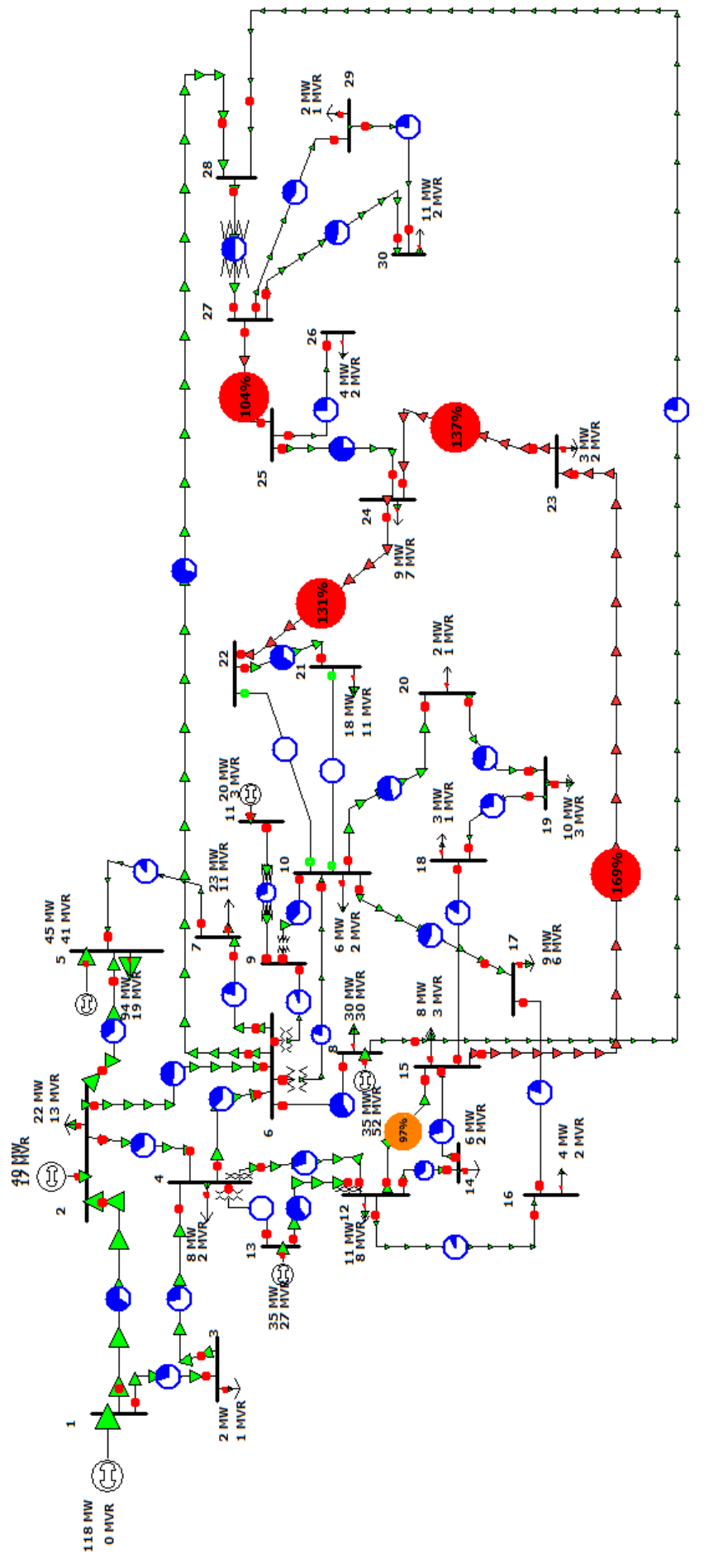


- Forced outage of line L31, released stress in the grid which is clearly reflected in Figures 9 and 10 with green colour. Figure 11 provides status of power flow in IEEE 30 bus system after forced outage of line L31. The temporary survival of this forced outage which is shown in Figure 11 can be handled by providing extra load, till the line between the buses is restored.

Figure 9 Maximum power angle analysis under perturbed grid (see online version for colours)

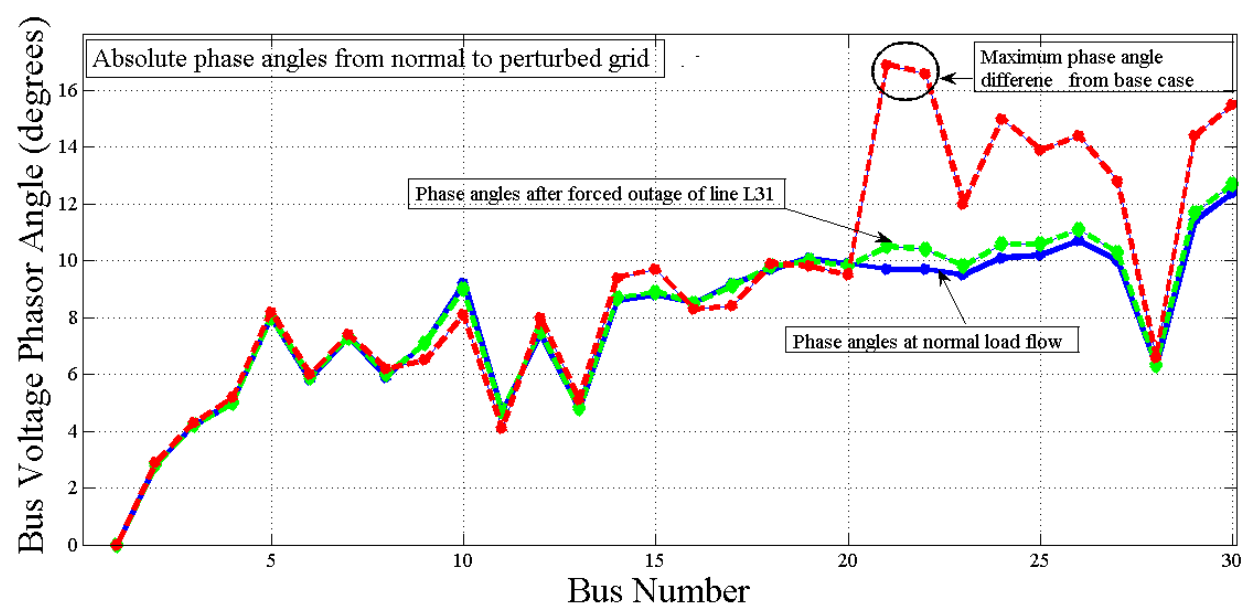

Figure 10 Power flow on transmission lines from base case to perturbed case and after forced line outage (see online version for colours)

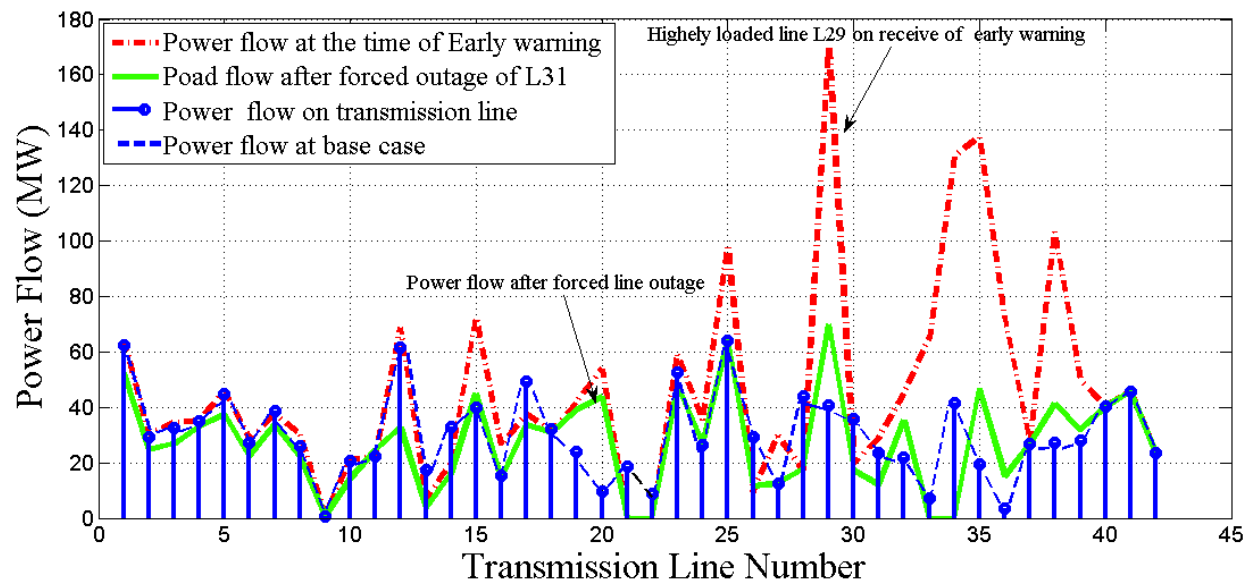


Figure 11 Load flow after forced line outage (see online version for colours)

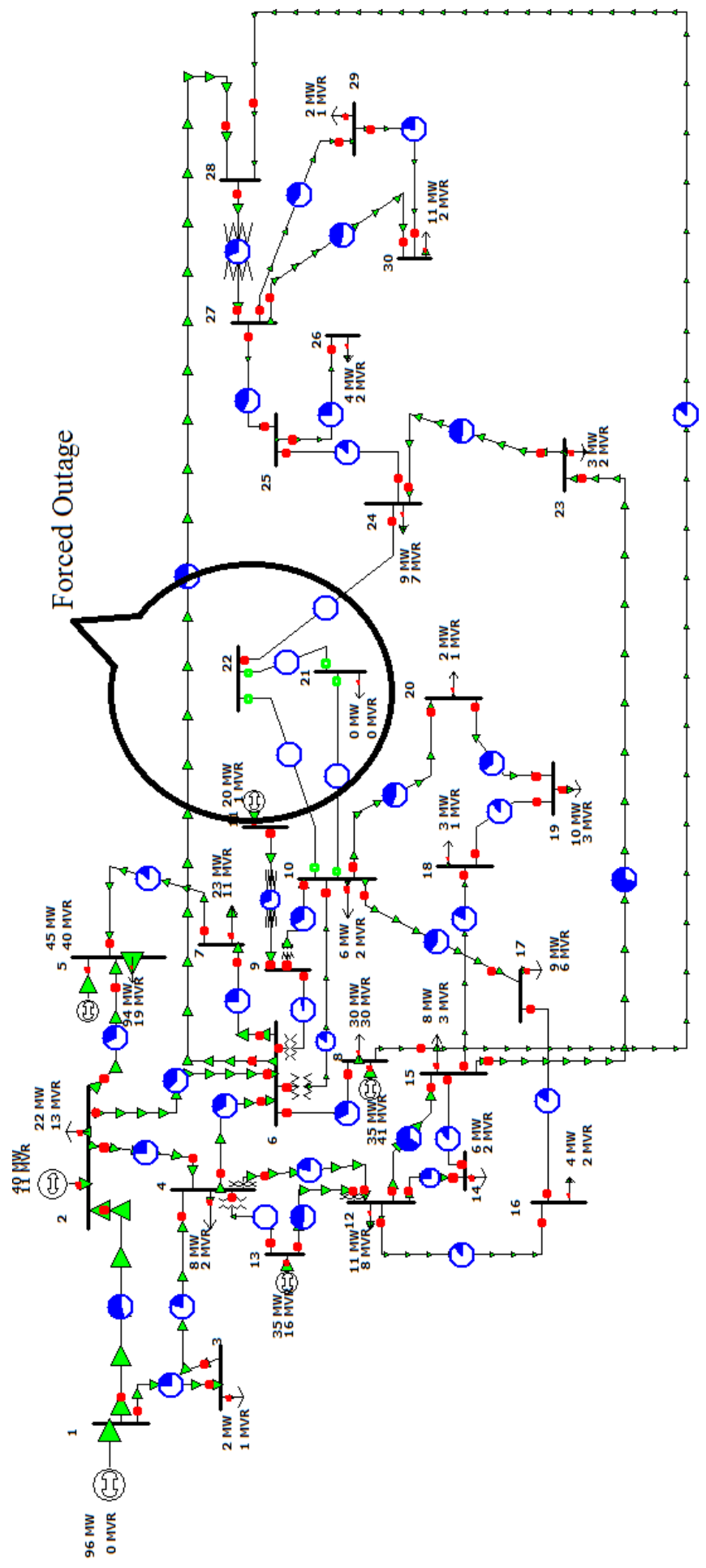




\section{Conclusions}

This paper is an attempt to propose a blackout prediction and mitigation model for upcoming WAMPAC technologies. The proposed model can be used for proactive cascade prediction and mitigation in planning of smart grid early warning system. The proposed model can be generalised with the higher order bus system or with real-time system. This work can be further extended for smart relays which can help for load shedding or controlled (pre-planned) islanding on prediction of blackout. The future scope of the proposed model is in the field of real world complex power networks that demands real-time contingency analysis with the robust control system and self-healing.

\section{Acknowledgements}

This work was supported in part by the Center of Excellence in Complex and Nonlinear Dynamical Systems, Veermata Jijabai Technological Institute, Mumbai, India, under TEQIP-II subcomponent (1.2.1).

\section{References}

'The enquiry committee on grid disturbance in Northern region on 30th July 2012 and in Northern, Eastern and North-Eastern region on 31st July 2012', New Delhi [online] htttp://www.cea.nic.in/reports/articles/god/grid_disturbance_report.pdf (accessed 16 August 2012).

Andersson, G., Donalek, P., Farmer, R., Hatziargyriou, N., Kamwa, I., Kundur, P., Martins, N., Paserba, J., Pourbeik, P., Sanchez-Gasca, J., Schulz, R., Stankovic, A., Taylor, C. and Vittal, V. (2005) 'Causes of the 2003 major grid blackouts in North America and Europe, and recommended means to improve system dynamic performance', IEEE Trans. on Power Systems, November, Vol. 20, No. 4, pp.1922-1928.

Cirio, D. and Lucarella, D. (2011) 'Wide area monitoring in the Italian power system: architecture, functions and experiences', Trans. on Elect. Power, May, Vol. 21, No. 4, pp.1541-1556.

Cummings, R.W. (2005) 'Predicting cascading failures', Presentation at NSF/EPRI Workshop on Understanding and Preventing Cascading Failures in Power Systems, Westminster CO, October.

De La Ree, J., Centono, V., Thorp, J.S. and Phadke, A.G. (2010) 'Synchronized phasor measurement applications in power systems', IEEE Trans. on Smart Grid, June, Vol. 1, No. 1, pp.20-27.

Denegri, G.B., Invernizzi, M. and Milano, F. (2002) 'A security oriented approach to PMU positioning for advanced monitoring of a transmission grid', in Proc. IEEE Int. Conf. Power Systems Technology, pp.798-803.

Ding, L., Gonzalez-Longatt, F.M. and Wall, P. (2013) 'Two step spectral clustering controlled islanding algorithm', IEEE Trans. Power Syst., Vol. 28, No. 1, pp.75-84.

Dobson, I., Parashar, M. and Carter, C. (2010) 'Combining phasor measurements to monitor cutset angles', 43rd Hawaii International Conference on System Sciences, January.

Durand, P. (2013) 'Technical and economic concepts related to the smart grid, a guide', A Review and Synthesis of Research on Smart Grid Benefits and Costs, 8 October.

Gnanadass, R. (2011) Appendix - A Data for IEEE-30 Bus Test System [online] https://www.google.com/shodhganga.inflibnet.ac.in/bitstream/10603/1221/18/ 18_appendix.pdf (accessed 7 April 2013). 
Gupta, S., Kazi, F., Wagh, S. and Kambli, R. (2015) 'Neural network based early warning system for an emerging blackout in smart grid power networks', Intelligent Distributed Computing, pp.173-183, Springer International Publishing, Switzerland.

Gupta, S.R., Kazi, F.S., Wagh, S.R. and Singh, N.M. (2014) 'Probabilistic framework for evaluation of smart grid resilience of cascade failure', IEEE Innovative Smart Grid Technologies Int. Conf. (ISGT), Asia, Kuala Lumpur, Malaysia, 20-23 May, pp.255-260.

Kamwa, I. and Grondin, R. (2002) 'PMU configuration for system dynamic performance measurement in large multiarea power systems', IEEE Trans. Power Syst., May, Vol. 17, No. 2, pp.385-394.

Kim, T.T. and Poor, H.V. (2011) 'Strategic protection against data injection attacks on power grids', IEEE Trans. on Smart Grid, June, Vol. 2, No. 2, pp.326-333.

Li, F., Qiao, W., Sun, H., Wan, H., Wang, J., Xia, Y., Xu, Z. and Zhang, P. (2010) 'Smart transmission grid: vision and framework', IEEE Trans. Smart Grid, September, Vol. 1, No. 2, pp.168-177.

Lu, X., Wang, W. and Ma, J. (2013) 'An empirical study of communication infrastructures towards the smart grid: design, implementation, and evaluation', IEEE Trans. on Smart Grid, March, Vol. 4, No. 1, pp.170-183.

Manousakis, N.M., Korres, G.N. and Georgilakis, P.S. (2012) 'Taxonomy of PMU placement methodologies', IEEE Trans. on Power Systems, May, Vol. 27, No. 2, pp.1070-1077.

Nuqui, R.F. and Phadke, A.G. (2005) 'Measurement unit placement techniques for complete and incomplete observability', IEEE Trans. Power Del., October, Vol. 20, No. 4, pp.2381-2388.

Sauter, T. and Lobashov, M. (2011) 'End-to-end communication architecture for smart grids', IEEE Trans. Ind. Electron., April, Vol. 58, No. 4, pp.1218-1228.

Siano, P., Cecati, C., Yu, H. and Kolbusz, J. (2012) 'Real time operation of smart grids via FCN networks and optimal power flow', IEEE Trans. Ind. Inf., November, Vol. 8, No. 4, pp.944-952.

Song, H., Wu, J. and Wu, K. (2014) 'A wide-area measurement systems-based adaptive strategy for controlled islanding in bulk power systems', Energies, April, Vol. 7, No. 4, pp.1852-2739, DOI: 10.3390/en7042631 [online] http://www.mdpi.com/jurnal/energies (accessed 22 September 2014).

Sullivan, R.L. and Lee, R. (1977) Power System Planning, McGraw-Hill International Book Company, New York.

Sun, K., Hur, K. and Zhang, P. (2011) 'A new unified scheme for controlled power system separation using synchronized phasor measurements', IEEE Trans. Power Syst., August, Vol. 26, No. 3, pp.1544-1554.

Wang, X-F., Song, Y. and Irving, M. (2008) Modern Power Systems Analysis, Springer Science + Business Media, LLC, New York, NY, USA.

$\mathrm{Xu}$, G., Vittal, V. and Meklin, A. (2011) 'Controlled islanding demonstrations on the WECC system’, IEEE Trans. on Power Syst., Vol. 26, No. 1, pp.334-343.

Yang, Q., Barria, J.A. and Green, T.C. (2011) 'Communication infrastructures for distributed control of power distribution networks', IEEE Trans. Ind. Inf., May, Vol. 7, No. 2, pp.316-327.

Zhabelova, G. and Vyatkin, V. (2012) 'Multi-agent smart grid automation architecture based on IEC 61850/61499 intelligent logical nodes', IEEE Trans. Ind. Electron., May, Vol. 59, No. 5, pp.2351-2362.

Zhang, P., Li, F. and Bhatt, N. (2010) 'Next-generation monitoring, analysis, and control for the future smart control center', IEEE Trans. on Smart Grid, September, Vol. 1, No. 2, pp.186-192. 